\title{
Panorama del manejo de malezas en cultivos de banano en el departamento de Magdalena, Colombia
}

\section{Outlook of weeds management in banana crops in the Magdalena department, Colombia}
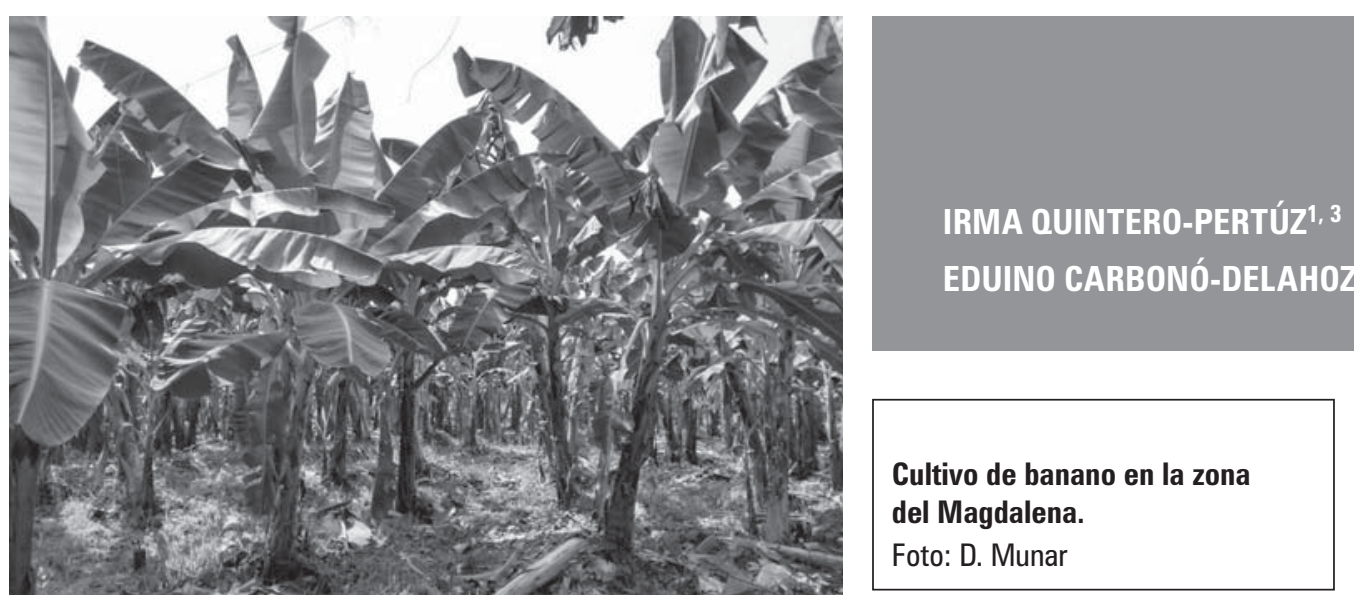

\section{RESUMEN}

En cultivos de banano del departamento del Magdalena el manejo de malezas se realiza, principalmente, mediante la aplicación de herbicidas, cuyo uso intensivo y continuo ha generado la contaminación de fuentes hídricas, sedimentos y ecosistemas marinos, afectando la biodiversidad y la salud humana. Ante la creciente aparición de malezas resistentes a herbicidas y la presión pública por reducir el uso de plaguicidas, es necesario ajustar las técnicas y métodos de control para incrementar la producción, mejorar la calidad de los productos y el bienestar de los agricultores, en armonía con el medio ambiente. Un nuevo enfoque que conciba al cultivo como un agroecosistema y a las malezas como integrantes del mismo, precisa conocer su biología y ecofisiología, así como, la interferencia y pérdidas que ocasionen. En este artículo se describe la situación actual del manejo de las malezas en plantaciones de banano y se discute la necesidad de promover el desarrollo de programas de investigación en malherbología que fundamenten la adopción de técnicas de manejo eficaces y ambientalmente aceptables.

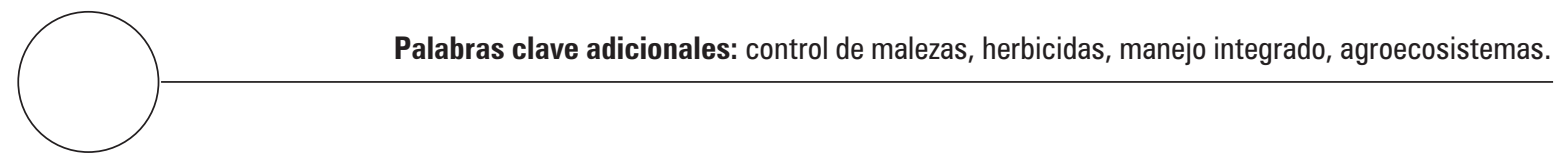

\footnotetext{
Facultad de Ingeniería, Universidad del Magdalena, Santa Marta (Colombia).

2 Herbario UTMC, Universidad del Magdalena, Santa Marta (Colombia).

3 Autor para correspondencia. irquipe@gmail.com
} 


\section{ABSTRACT}

In banana crops in the Magdalena department, weed management is primarily done through the application of herbicides, whose intensive and continuous use has led to the contamination of water sources, sediment and marine ecosystems, affecting the biodiversity and human health. Given the increasing occurrence of herbicide-resistant weeds and public pressure to reduce pesticide use, it is necessary to adjust the control techniques and methods in order to increase production and improve the product quality and welfare of farmers in line with the environment. A new approach that sees the crop as an agroecosystem and weeds as a part of it needs to understand the biology and ecophysiology, as well as the resulting interference and losses. In this article, the current situation of weed management is described for banana plantations along with the need to promote the development of research programs in weed science that will contribute to the adoption of techniques that are effective and environmentally acceptable.

Additional key words: weed control, herbicides, integrated management, agroecosystems.

Fecha de recepción: 15-07-2015

Aprobado para publicación: 20-11-2015
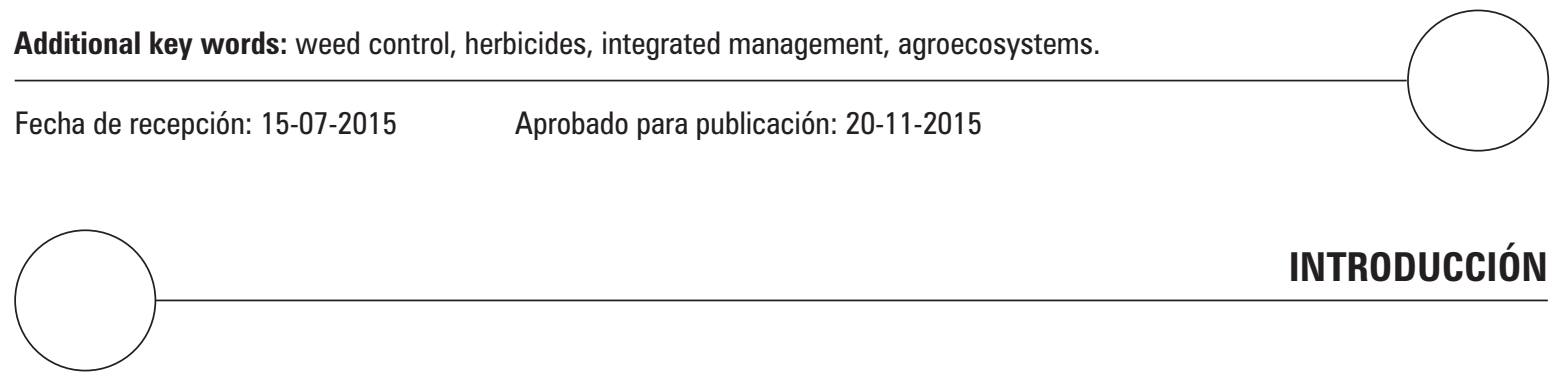

INTRODUCCIÓN

Las malezas pueden convertirse en las principales limitaciones bióticas de la producción agrícola en los países en desarrollo (Mortimer, 1997), en donde es característico no prestarles la adecuada atención, debido al escaso conocimiento de aspectos relativos a la interferencia y a la ausencia de programas nacionales de investigación sobre su ecobiología y control (Labrada, 1997). A pesar de los avances alcanzados en el control por métodos químicos, se contemplan con preocupación, efectos contradictorios por la aparición de biotipos resistentes a herbicidas (Guglielmini et al., 2007; Vencill et al., 2012), la contaminación de aguas superficiales y subterráneas (Bedmar, 2006), el incremento de la erosión del suelo (Del Valle et al., 2010), la residualidad en alimentos (FAO, 2013), esto último con implicaciones directas sobre la competitividad de los productos agrícolas en los mercados internacionales. Los problemas ambientales de la agricultura, sumados a la crisis alimentaria actual, han impuesto el reto de reducir el uso de insumos agrícolas externos en la perspectiva de una producción sostenible (Labrada, 1997). Desde un punto de vista ecológico, muchas de las faenas agrícolas que representan disturbios del ecosistema y cambian la disponibilidad de recursos, substrato o ambiente (Menalled, 2010), también deben ser mejorados en términos de eficiencia y efectividad para reducir sus impactos económicos y ambientales.

En el departamento de Magdalena, la agricultura es una de las actividades económicas básicas y el problema de las malezas es evidente. Por una parte, afectan la producción de los cultivos, y por otra, para su control se usa una gama de moléculas según el problema o la especie (Bonilla et al., 2000). En la mayoría de los casos, el manejo inadecuado causa contaminación de fuentes hídricas, sedimentos y ecosistemas marinos, que afectan la biodiversidad y la salud de las personas (PNUMA-UCR/CAR, 2012). Parte de ello es generado por el cultivo de banano, principal producto de exportación del departamento desde los primeros años del siglo XX, que hoy ocupa un lugar destacado en la economía regional por el área cultivada, la generación de empleo y de divisas (Viloria, 2008). Urge entonces, mejorar las 
medidas para controlar las malezas en armonía con el medio ambiente, como una de las vías para incrementar la producción, mejorar la calidad de los productos y el bienestar de los agricultores.

El objetivo de la presente revisión es describir la situación del control de malezas en plantaciones bananeras y discutir la necesidad de promover nuevos enfoques técnicos que sean no solo económicamente eficaces, sino también ecológica y socialmente aceptables.

\section{IMPORTANCIA DE LAS MALEZAS EN LOS SISTEMAS AGRÍCOLAS}

Con el término "maleza" se quiere significar toda planta que crece en donde no se desea, plantas "fuera de lugar" o "plantas indeseables" (Monaco et al., 2002). En general, expresa una noción de nocividad por su intención conceptual, mas no corresponde a una realidad natural (Dekker, 2011), ya que no existe ningún atributo morfológico o fisiológico que permita caracterizar a una especie vegetal como maleza (Sabbatini et al., 2004). Que se catalogue o no a una planta como mala hierba, depende de la opinión del observador. Así, la aversión de los agricultores por estas plantas, es debida al impacto negativo que ocasionan sobre el rendimiento de los cultivos, al competir por los nutrientes, el agua y la luz, perjudicar la cantidad y calidad de la producción agrícola, interferir en las labores de cosecha (Menalled, 2010), y hospedar patógenos causantes de enfermedades e insectos plagas (Culliney, 2005).

Los daños causados por las malas hierbas sobre la producción agrícola mundial, se han estimado en 13,2\% o US\$75,6 miles de millones por año (Oerke et al., 1994), aunque de manera general, se acepta que ocasionan una disminución de la producción entre $30 \%$ y $50 \%$ (Plaza et al., 2006). Sin embargo, tales pérdidas o daños varían según los países, regiones del mundo y cultivos afectados (Swanton et al., 1993; Pimentel et al., 2001). En Colombia, la estimación de pérdidas ocasionadas por las malezas en los principales cultivos, sin incluir banano, fue 35\% (CIAT, 1989). Para este cultivo, no hay estimaciones actualizadas de pérdidas ocasionadas por malezas, pero en general se aceptan como un problema cuyo control incide en los costos totales de producción. Para 1999, Augura fijó la participación en la estructura de costos del control químico de malezas en 1,25\%, incluyendo mano de obra e insumos (Pinilla y García, 2002). En plantaciones de exportación del Urabá, Martínez y Hoyos (2012) reportaron costos relacionados con el control de malezas por US\$166,6/hectárea, lo que equivale a $5,4 \%$ de los costos totales del manejo agronómico. En la zona bananera del Magdalena, se calcula que los costos atribuidos al control de malezas equivalen a $5,7 \%$ de los costos directos de precosecha (MADR, 2005).

Entre los efectos de la interferencia de las malezas en banano, se han reportado: reducción de altura, grosor del pseudotallo y peso de racimo (Plaza, 2012); deficiencia de nitrógeno por efecto de la competencia, reflejada en amarillamiento del follaje joven (Terry, 1997), crecimiento lento con pocos hijos, retraso de la floración con el consecuente alargamiento del ciclo del cultivo, disminución del rendimiento. Además, se dificultan labores como la aplicación de fertilizantes, el desmache, el deshoje, la cosecha (Pinilla y García, 2002). En banano y plátano, se reconoce un periodo crítico de competencia de las malezas durante el establecimiento de los cultivos (Terry, 1997). Un buen control durante este periodo mejora la eficiencia en el uso de fertilizantes, acelera el crecimiento y aumenta el rendimiento (Seeyave y Phillips, 1970; Badgujar et al., 2003). Un segundo periodo de competencia es señalado por Plaza (2012) entre el brote de la flor y el engrosamiento de los frutos. Prameela (2010) reportó como etapas críticas, las de la fase vegetativa temprana de crecimiento hasta los tres y seis meses después del trasplante y la época de desarrollo del racimo, en las que el rendimiento se ve afectado. Para la zona bananera de Magdalena, no se ha reportado un periodo crítico de competencia, ni el efecto verdadero que pueda tener la 
duración de los intervalos de desyerbe (Pinilla y García, 2002).

Terry (1997) destaca que las malezas más agresivas asociadas al cultivo de banano y plátano son gramíneas, entre las cuales sobresalen: Axonopus compressus, Cynodon dactylon, Digitaria abyssinica, Imperata cylindrica, Panicum maximum, Paspalum scrobiculatum, Paspalum conjugatum, Pennisetum purpureum, y la Cyperaceae: Cyperus rotundus. Sobre las de hoja ancha, señala que estas no compiten con el cultivo, aunque algunas pueden ser problemáticas como Chromolaena odorata, Convolvulus spp. e Ipomoea spp. Para las zonas bananeras de Urabá y Magdalena, Pinilla y García (2002) reportaron 41 especies de importancia económica distribuidas en 20 familias, entre las cuales las más diversificadas fueron las familias Poaceae, Cyperaceae y Commelinaceae. Para el Magdalena, Carbonó y Cruz (2005) identificaron 202 especies asociadas al cultivo y como familias más diversificadas: Poaceae (38 especies), Fabaceae (27) y Asteraceae (11), sin discriminar por el nivel de importancia económica. Especies de la familia Poaceae, fueron reconocidas como las más nocivas para el banano en esta zona (Moreno et al., 2009).

\section{MÉTODOS DE CONTROL}

Para controlar malezas en banano se aplican los métodos cultural, mecánico y químico. Este último es el más usado en la mayoría de las plantaciones comerciales. Sin embargo, los condicionantes impuestos por normas del comercio internacional de productos agrícolas (Izquierdo y Rodríguez, 2006; Cenckowsky y Berger, 2010), han obligado al uso de otras prácticas (Bonilla et al., 2000), aunque estas no alcanzan una generalización en el departamento.

\section{Control cultural}

Se destaca el uso de cubiertas sobre el suelo durante el desarrollo del cultivo, utilizando restos de cosecha y procurando no cubrir el área de plateo para facilitar las labores de fertilización y desmache, o plantas vivas como coberturas (Pinilla y García, 2002). Para la región, se identificaron como especies apropiadas Teramus volubilis, Callisia cordifolia, Desmodium scorpiurus y $D$. triflorum, seleccionadas entre arvenses asociadas al cultivo (Carbonó y Cruz, 2005). C. cordifolia, ha sido establecida con relativo éxito en algunas plantaciones, aunque no hay evaluaciones de los efectos de esta práctica sobre el control de malezas, el suelo y la producción del cultivo.

\section{Control manual y mecánico}

La limpieza manual, denominada "plateo", se hace con machete sobre la unidad de producción en un radio de $1 \mathrm{~m}$ a partir del pseudotallo. El control mecánico, denominado "chapia", se efectúa con guadaña y consiste en la poda de las malezas en las calles a una altura de cinco centímetros, evitando descubrir totalmente el suelo. La limpieza se mantiene durante el primer año de establecido el cultivo, iniciando a las cuatro semanas de la siembra, realizada en ciclos calendario de 4 a 6 semanas, dependiendo de las condiciones climáticas y tipo de malezas prevalentes (Moreno et al., 2009). Se consideran métodos eficaces para el control de la mayoría de las especies asociadas al cultivo, especialmente las de hoja ancha (Pinilla y García, 2002), y los más usados por pequeños productores, aunque representan un alto costo por el requerimiento de mano de obra.

\section{Control químico}

Es el más utilizado en la mayoría de las plantaciones bananeras y solo hasta hace muy poco tiempo se ha intentado racionalizar su uso en la región. Se permiten los herbicidas aprobados por el Departamento de Agricultura de Estados Unidos (USDA) y por la Agencia de Protección Ambiental (EPA). Si bien existe una amplia gama de productos en el comercio, los productores, asistentes técnicos y administradores de fincas generalizaron el uso del Paraquat y Glifosato, con 
aplicaciones oscilantes entre 4 y 6 ciclos por ha/ año (Bonilla et al., 2000). Según PNUMA-UCR/ CAR (2012), para Magdalena, se registraron aplicaciones de glifosato en dosis de $100 \mathrm{~mL} / 20 \mathrm{~L}$ de agua para el control de malezas gramíneas, y de Paraquat en dosis de $150 \mathrm{~mL} / 20 \mathrm{~L}$ de agua para las de hoja ancha. Sin embargo, desde hace cuatro años, el Paraquat fue retirado de la explotación bananera por exigencia del mercado europeo donde el agroquímico tiene restricciones por su categoría toxicológica (Aguirre-Buitrago et al., 2014), por lo que el control químico quedó supeditado casi exclusivamente a aplicaciones de Glifosato, con aumento de la dosis hasta $200 \mathrm{~mL} / 20$ L de agua en algunas fincas, para controlar las más agresivas en los periodos considerados críticos. De acuerdo con información de técnicos y administradores de fincas, algunas especies han creado resistencia a los productos usados; lo que merece especial atención, porque repetidas aplicaciones de herbicidas con similar modo de acción imponen una presión de selección que lleva a la resistencia, en especies comúnmente susceptibles (Radosevich et al., 1997). Debe considerarse que a nivel mundial, desde 1970 hay un incremento anual estimado en nueve nuevos casos de poblaciones de malezas resistentes a herbicidas, con una tendencia de aumento continuo a pesar de los esfuerzos por educar a los agricultores sobre cómo manejar la resistencia (Shaner, 2014).

\section{CONOCIMIENTOS BÁSICOS PARA EL MANEJO INTEGRADO DE MALEZAS}

A pesar de los esfuerzos y tecnologías desarrolladas para su control, las malezas no han podido ser erradicadas eficazmente (Smith et al., 2006), ello es atribuido a su adecuada adaptación biológica a entornos perturbados (Dekker, 2011). El manejo de los cultivos constituye la mayor fuerza selectiva en la evolución de las malezas (Neve et al., 2009) y determina las características de las comunidades vegetales presentes al modificar su abundancia y composición (Menalled et al.,
2001; Davis et al., 2005), afectar su distribución espacial y su capacidad competitiva (Pollnac et al., 2008; Smith et al., 2010) como consecuencia de un proceso de co-evolución entre cultivos y malezas asociadas (Guglielmini et al., 2007). En los últimos años, el uso indiscriminado de pesticidas sintéticos en los cultivos, no solo afectó a los agentes objetivo, sino que también causó daños no intencionales a otros no objetivo, incidiendo en la disminución constante de la biodiversidad de los sistemas agrícolas (Huang y Chou, 2005). Con los herbicidas, la diversidad de especies puede variar en función del número de aplicaciones y del tipo de molécula aplicada, y generar cambios en las plantas atribuibles a la resistencia natural de las especies comunes al producto o a evolución de la resistencia de la población (Owen y Zelaya, 2005).

En el mundo se han reportado 1.185 biotipos de 246 especies de malezas resistentes a herbicidas, lo que representa un incremento de 38 nuevas especies desde 2012 (Harker y O'Donovan, 2013; Heap, 2015); de estas, 26 son malezas frecuentes en cultivos de banano de Magdalena. Actualmente se identifican 32 especies resistentes a Glifosato en el mundo (Heap, 2015), de las cuales 7 crecen en plantaciones bananeras en Magdalena: Amaranthus spinosus, Conyza canadensis, Digitaria insularis, Echinochloa colona, Eleusine indica, Leptochloa virgata y Sorghum halepense; así mismo, hay 29 especies con resistencia al Paraquat (Heap, 2015), y cuatro de ellas son comunes en esta región: Ischaemum rugosum, Eleusine indica, Solanum americanum y Conyza canadensis. Es destacable que E. indica aparece resistente a Glifosato y a Paraquat. La pérdida o reducción en la eficacia de los herbicidas se convierte en un problema a resolver en la búsqueda de métodos alternativos, viables para el control de las malezas.

La preocupación actual por la conservación del medio ambiente, consiste en no encontrar métodos de control efectivos de plantas no deseadas sin afectar la producción de cultivos, el ambiente y los organismos benéficos. A menos que se en- 
tienda los componentes del agroecosistema y sus complejas interacciones en los entornos físicos y químicos, es difícil predecir el mejor método de producción agrícola sin efectos perjudiciales (Huang y Chou, 2005). Al respecto Altieri (1999) argumenta que un enfoque agroecológico se constituye en el fundamento científico de la agricultura sostenible, ya que brinda conceptos, características y principios ecológicos para analizar, diseñar, administrar y conservar los recursos de los sistemas agrícolas toda vez que los define, clasifica y estudia desde una perspectiva agronómica, ecológica, socioeconómica, étnica y sociocultural.

Para un mejor entendimiento del comportamiento de las malezas en el diseño de estrategias adecuadas de manejo, Labrada y Parker (1996) han considerado cuatro componentes básicos:

\section{Identificación de las malezas y su nivel de infestación}

La caracterización de la comunidad de plantas asociadas al cultivo es una herramienta básica para su adecuado manejo (Gámez et al., 2011; Ariza y Almanza-Merchán, 2012). Una correcta identificación taxonómica cumple un papel fundamental para conseguir las recomendaciones en el desarrollo de un programa de manejo y errores taxonómicos pueden derivar en una subestimación del problema o en el uso de medidas de control equivocadas (Fernández, 1982), con consecuencias negativas sobre la abundancia relativa y distribución de la población de plantas (Bortolus, 2008), dado que el complejo de malas hierbas tiene una dinámica de variación que va desde la introducción de especies foráneas, hasta la aparición de ecotipos. En los procesos de identificación, los catálogos ilustrados de malezas comunes y guías de reconocimiento de plántulas son necesarios para el desarrollo de programas de manejo, como fuen- te de información para agricultores, comunidades locales y técnicos, con aplicación en procesos de investigación y educación. Actualmente no se cuenta con catálogos de malezas para la región, por lo que su elaboración a partir de un inventario actualizado debe ser considerada en los futuros programas de investigación.

\section{Biología y ecofisiología de las especies de malezas prevalentes}

Una mejor comprensión de la biología de las malezas, permite conocer sus características morfológicas y fisiológicas, sus ciclos de vida, etapas fenológicas y hábitos de crecimiento, fundamental para determinar sus estadios más vulnerables y sus épocas de aparición en el campo (Campbell y Grice, 2000); así como sus medios de propagación, aspectos fisiológicos de la germinación de semillas y de la reproducción vegetativa con el estudio del banco de semillas del suelo (Bhowmik, 1997), cuya importancia estriba en que se constituye en el historial de las plantas en la región y a largo plazo determina el futuro de las poblaciones (Ball, 1992). Este conocimiento ecológico da la posibilidad de un mejor entendimiento de las dinámicas de población, indispensable al momento de definir operaciones de manejo (Golafshan y Yasari, 2012; Schwartz et al., 2015). Se ha señalado que la baja adopción de programas de Manejo Integrado de Malezas (MIM) obedece a la falta de conocimientos sobre las bases agroecológicas (Labrada y Parker, 1996). A la vez, el difícil control de las variables ambientales y biológicas que determinan la abundancia e impacto de las malezas disminuye el valor predictivo de la información ecológica. Aunque, el prolongado periodo de tiempo asociado a la investigación ecológica de las malezas, va en contravía de las necesidades apremiantes de los agricultores, el éxito de un MIM requiere de la ampliación de las bases del conocimiento agroecológico básico y aplicado (Menalled, 2010). 


\section{Efectos competitivos de las especies de malezas prevalentes}

Está establecido que las malezas causan la mayoría de daños a los cultivos durante ciertas etapas del crecimiento, lo que se ha denominado periodo crítico de competencia, y el conocimiento de este periodo es especialmente importante para intervenir y prevenir pérdidas del rendimiento (Knezevic y Datta, 2015). El grado de interferencia maleza-cultivo depende tanto de factores relacionados con el cultivo mismo, como la variedad, el distanciamiento y densidad de siembra, así como de las malezas en cuanto a especie, densidad y distribución, además de la época y duración de la interferencia. Sin embargo, todos estos factores pueden cambiar según las condiciones climáticas, edáficas, nutricionales y de manejo (Martins y Pitelli, 1994; Evans et al., 2003); en tal sentido, se debe conocer las particularidades en cada caso.

\section{Estrategias de control técnicamente efectivas, económicamente viables y seguras para el medio ambiente}

Mortimer (1997) clasifica las opciones de manejo que tienen los agricultores en dos categorías: componentes agronómicos y de intervención; entre los primeros destaca: preparación del terreno, método para establecer el cultivo, variedades competitivas, asociación de cultivos, normas de semillas, pureza de las semillas del cultivo, manejo del agua, manejo de los fertilizantes, rotación de cultivos y diversidad de cultivos; y entre los segundos: acolchado, cultivación del terreno, desyerbe manual, desyerbe dirigido, desyerbe químico, control biológico, momento y frecuencia de la intervención.

Para el cultivo de banano en la región del Magdalena se han propuesto un conjunto de Buenas Prácticas Agrícolas (BPA), orientadas hacia una producción sostenible en respuesta a la necesidad de mitigar la degradación del medio ambiente costero y marino de la región, causado por plaguicidas de uso agrícola (Moreno et al., 2009). Entre estas labores se incluyen las referidas a un manejo integrado de malezas, donde se sugiere combinar los diferentes métodos de control recomendados (químico, cultural, físico y mecánico). Cabe señalar que en las fincas modelo donde se implementaron las BPA para control de malezas, se redujo el consumo de herbicidas, hasta 100\% en una de ellas, donde el manejo se realizó con coberturas vivas, disposición adecuada de los residuos de cosecha como cubierta y control manual de las más agresivas (PNUMA-UCR/CAR, 2012). Aunque es un avance importante, es preciso hacer una valoración global, para medir el impacto económico, social y ambiental, real en el área de cultivo, tanto en fincas de pequeña como de gran extensión, considerando involucrar a todos los productores y determinar la eficiencia y eficacia de estos métodos en el mediano y largo plazo.

\section{NECESIDADES DE INVESTIGACIÓN SOBRE MALEZAS EN LA REGIÓN}

Fuentes y Romero (1991) resaltaron la necesidad de desarrollar estudios en aspectos de sistemática, biología, ecología, interferencia de malezas, sistemas de manejo en cultivos y de impacto ambiental de los herbicidas para mejorar la investigación en las ciencias de las malezas en Colombia. Aunque hay avances en algunos cultivos y especies de malezas (Plaza et al., 2006, Zamorano et al., 2008; Canal et al., 2009), no ha sido notorio en plantaciones de banano.

En el departamento de Magdalena no se conocen programas básicos de investigación en malherbología. En banano, la escasa investigación la siguen realizando algunas casas comerciales de agroquímicos y se reduce a evaluar dosis y mezclas de productos o el uso de nuevas moléculas. El Centro de Investigaciones del Banano (Cenibanano), que desarrolla investigación en la línea de fitoprotección, se centra en el manejo de la sigatoka negra, y por extensión, brinda asesoría a 
cultivadores, principalmente en el uso y manejo adecuado de herbicidas.

El programa de Ingeniería Agronómica de la Universidad del Magdalena, a pesar de que contempla en su plan curricular un curso obligatorio de malherbología, tiene pocos trabajos realizados en este campo, y los desarrollados se relacionan con el reconocimiento de flora asociada a los cultivos, evaluación de dosis de herbicidas y más recientemente, con la identificación de especies promisorias como coberturas, sin que obedezcan a un plan definido de estudio en el campo de la ciencia de las malezas. Estudios de biología, ecología y dinámica poblacional, no han sido abordados como un componente importante para comprender su comportamiento y mejorar su manejo. Se sabe muy poco sobre el banco de semillas del suelo, de la interferencia, periodos críticos de competencia, el monitoreo regular de las infestaciones y las pérdidas posibles de cosecha. El poco conocimiento de estos aspectos constituye una limitante para el desarrollo de un manejo de malezas adecuado en la región, lo cual ha sido propiciado, quizás, por falta de conciencia por parte de los agricultores y entes gubernamentales acerca de las pérdidas reales que ocasionan. La ausencia de investigaciones locales en alternativas de manejo y la falta de servicios de extensión, son otros factores que también han limitado el desarrollo de estos programas.

Dada la creciente aparición de malezas resistentes a herbicidas y la consistente presión pública para reducir el uso de plaguicidas, se requiere, con urgencia, un verdadero manejo integrado de malezas (Harker y O'Donovan, 2013). Para lograr ese cambio de paradigma, es preciso concientizar, no solo a agricultores y técnicos, sino también a investigadores y entes financiadores de investigación, sobre la necesidad de pasar de la interminable repetición de los mismos experimentos en diferentes cultivos, malezas o herbicidas, a la generación de tecnologías basadas en investigación científica (Ward et al., 2014). Un programa de investigación en malherbología, debe partir del conocimiento de la biología de las malezas, de un enfoque integrado para su manejo, y de la educación de investigadores, productores y técnicos sobre la importancia y amplitud de esta ciencia (Breen y Ogasawara, 2011). La integración con otras disciplinas de la biología no debe subestimarse, pues se ha evidenciado que ello propicia la expansión de la investigación más allá del enfoque limitado al control químico, para enfatizar el manejo integrado basado en principios biológicos y ecológicos, en el que las malezas se pueden evaluar como un componente integral, donde además de tener en cuenta sus impactos negativos, deben analizarse también los factores causales de su abundancia y función ecológica. Así, la comprensión de las dinámicas de interferencia (competencia y alelopatía) de las malezas dentro de un enfoque ecofisiológico puede ayudar a los fitomejoradores a producir variedades de cultivo competitivas (Belz, 2007). Además, el potencial alelopático de algunas especies, puede contemplarse como una oferta potencial para el desarrollo de futuros herbicidas (Weston y Duke, 2003), por la capacidad de suprimir la germinación y el crecimiento, incluso en aquellas malezas resistentes a herbicidas (Sangeetha y Baskar, 2015).

También se considera que las malezas pueden proporcionar diferentes servicios ecológicos (Menalled, 2010), ayudando al control de la erosión del suelo, modificando procesos microclimáticos, impactando la dinámica hidrológica, constituyendo una reserva de germoplasma, alterando el ciclo de nutrientes y su retención en el suelo, y proporcionando refugio y alimento a los enemigos naturales de las plagas (Cruz et al., 2001; Jordan y Vatovec, 2004; Nicholls, 2006). Tales componentes no han sido medidos en la región, por lo que debe contemplarse su estudio para el desarrollo de programas de manejo integrado de malezas en banano. 


\section{CONCLUSIONES}

El control de malezas en plantaciones bananeras de Magdalena que se realiza, principalmente, con la aplicación de herbicidas, requiere de un nuevo enfoque. La evidente contaminación generada sobre fuentes hídricas, sedimentos y ecosistemas marinos, los efectos sobre la biodiversidad y la salud humana, así como las exigencias de los mercados internacionales, y la no menos importante aparición de algunas especies con resistencia a los productos de mayor uso, conducen a la necesidad de priorizar en el desarrollo de programas de manejo integrado de malezas y su adopción para una producción más limpia. Al respecto, es fundamental el análisis agroecológi- co sistemático de los mecanismos determinantes de la abundancia, dispersión e impacto de las malezas en cultivos de banano de la región.

En esta perspectiva, sería necesario fortalecer los departamentos de sanidad del sector productivo y generar alianzas con la academia para desarrollar programas de investigación en malherbología, que involucre a agricultores, técnicos, investigadores y estudiantes de agronomía en el estudio de la ecobiología de las malezas prevalentes, el periodo crítico de interferencia, pérdidas ocasionadas y alternativas de manejo. Conviene, también, vigorizar los programas de extensión que garanticen la validación y aplicación de los resultados de investigación obtenidos.

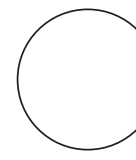

Aguirre-Buitrago, J. C., S. C. Narváez-González, M. E. Bernal-Vera y E. Castaño-Ramírez. 2014. Contaminación de operarios con clorpirifos, por práctica de "embolsado" de banano (Musa sp.) en Urabá, Antioquia. Revista Luna Azul 38, 191-217. Doi: 10.17151/luaz.2014.38.12

Altieri, M. A. 1999. Agroecología. Bases científicas para una agricultura sustentable. Editorial Nordan Comunidad, Montevideo.

Ariza, C.A. y P.J. Almanza-Merchán. 2012. Identificación y clasificación en biotipos de las malezas asociadas con el cultivo de la palma de aceite. Cienc. Agric. 9(2), 87-96

Ball, D.A. 1992. Weed seed bank response to tillage, herbicide and crop rotation sequence. Weed Sci. 40, 654-656.

Badgujar, C.D., S.M. Dusane y S.S. Deshmukh. 2003. Influencia de la frecuencia de deshierbe sobre el desempeño del banano "Basrai" (AAA). InfoMusa $12(2), 12-13$

Bedmar, F. 2006. Comportamiento ambiental de los herbicidas en el suelo: conceptos y resultados regionales. Seminario de Actualización Técnica "Manejo de Malezas". Serie Actividades de difusión N465. En: Sitio argentino de producción animal, http:// www.produccion-animal.com.ar; consulta: 8 de mayo de 2015.

\section{REFERENCIAS BIBLIOGRÁFICAS}

Belz, R. 2007. Allelopathy in crop/weed interactions - an update. Pest Manage. Sci. 63, 308-326. Doi: $10.1002 /$ ps.1320

Bhowmik, P. 1997. Weed biology: importance to weed management. Weed Sci. 45, 349-356.

Bonilla, J., J. Peinado, M. Urdaneta y E. Carrascal. 2000. Informe nacional sobre uso y manejo de plaguicidas en Colombia, tendiente a identificar y proponer alternativas para reducir el escurrimiento de plaguicidas al mar Caribe. Ministerio del Medio ambiente, Dirección General Ambiental Sectorial Proyecto PNUMA/CAR Global Environment Facility. Bogotá.

Bortolus, A. 2008. Error cascades in the biological science: the unwanted consequences of using bad taxonomy in ecology. Ambio 37(2), 114-118. Doi: 10.1579/0044-7447(2008)37[114:ECITBS]2.0.CO;2

Breen, J.y M. Ogasawara. 2011. A vision for weed science in the twenty-first century. Weed Biol. Manage. 11, 113-117. Doi: 10.1111/j.1445-6664.2011.00418.x

Campbell, S.D. y A.C. Grice. 2000. Weed biology: a foundation for weed management. Trop. Grassl. 34, 271-279.

Canal R., O. Arnaude, A. Domínguez, B. Valverde y C. Fuentes. 2009. Caracterización morfológica de poblaciones de arroz maleza, en el distrito de riego del 
Río Zulia, Norte de Santander, Colombia. Agron. Trop. 59(4), 387-400.

Carbonó, E. y Z. Cruz. 2005. Identificación de coberturas promisorias para cultivo de banano en la zona de Santa Marta, Colombia. Rev. Intrópica 2(1), 7-22.

Cenckowsky, U. y J. Berger. 2010. Generación de capacidad comercial hacia EFTA: Inteligencia de mercado para Colombia - Frutas y Verduras orgánicas. OSEC, Zurich/Proexport, Bogotá.

CIAT (Centro Internacional de Agricultura Tropical). 1989. Información básica sobre la competencia entre las malezas y los cultivos; guía de estudio para ser usada como complemento de la unidad audiotutorial sobre el mismo tema. $2^{\text {a }}$. ed. CIAT, Cali, Colombia.

Cruz, R., C. Rojas, H. Lobón y C. Burgos. 2001. El papel de las malezas en la reducción de la lixiviación de nutrimentos en cultivos de banano en el trópico húmedo. Manejo Integrado de Plagas 62, 29-37.

Culliney, T. 2005. Benefits of classical biological control for managing invasive plants. Crit. Rev. Plant Sci. 24, 131-150. Doi: 10.1080/07352680590961649.

Davis, A., K. Renner y K. Gross. 2005. Weed seed bank and community shifts in a long-term cropping systems experiment. Weed Sci. 53, 296-306. Doi: 10.1614/WS-04-182.

Dekker, J. 2011. Evolutionary ecology of weeds. Agronomy Department, Iowa State University, Ames, IA.

Del Valle, L., J. Busnelli y M. Sampietro. 2010. Incremento de erosión y suelos degradados por acciones antropogénicas y variaciones climáticas, Tucumán. Rev. Asoc. Geol. Argent. 66(4), 499-504.

Evans, S., S. Knezevic, J. Lindkquist, C. Shapiro y E. Blankenship. 2003. Nitrogen application influences the critical period for weed control in corn. Weed Sci. 51, 408-417. Doi: 10.1614/0043-1745(2003)051[0408:NAITCP]2.0. $\mathrm{CO} ; 2$

FAO. 2013. Pesticide residues in food 2012. FAO/WHO Meeting on Pesticide Residues. FAO, Roma.

Fernández, A. 1982. Manejo integrado de malezas. Planta Daninha 5(2), 69-79. Doi: 10.1590/S010083581982000200010

Fuentes, C. y C. Romero. 1991. Una visión del problema de las malezas en Colombia. Agron. Colomb. 8(2), 364-378.

Gámez, A., M. Hernández, R. Díaz y J. Vargas. 2011. Caracterización de la flora arvense asociada a un cultivo de maíz bajo riego para producción de jojotos. Agron. Trop. 61(2), 133-139.

Guglielmini, A., C. Ghersa y E. Satorre. 2007. Co-evolution of domesticated crops and associated weeds. Ecol. Austral 17, 167-178.

Golafshan, M.G. y E. Yasari. 2012. Comparison of sampling methods for estimating seed bank and weed population densities during the growing season. J. Agric. Sci. 4(9), 39-47. Doi: 10.5539/jas. v4n9p39

Heap, I. 2015. The international survey of herbicide resistant weeds. En: www.weedscience.org; consulta: 8 de junio de 2015 .

Harker, K. y J. O’Donovan. 2013. Recent weed control, weed management, and integrated weed management. Weed Technol. 27, 1-11. Doi: 10.1614/WTD-12-00109.1

Huang, H.C. y C.H. Chou. 2005. Impact of plant disease biocontrol and allelopathy on biodiversity and agricultural sustainability. Plant Pathol. Bull. 14, 1-12.

Izquierdo, J. y M. Rodríguez. 2006. Buenas Prácticas Agrícolas (BPA): En busca de sostenibilidad, competitividad y seguridad alimentaria. FAO, Santiago.

Jordan, N. y C. Vatovec. 2004. Agroecological benefits from weeds. pp. 137-158. En: Inderjit (ed.). Weed biology and management. Kluwer Academic Publishers, The Netherlands. Doi: 10.1007/978-94017-0552-3

Knezevic, S. y A. Datta. 2015. The critical period for weed control: Revisiting data analysis. Weed Sci. Spec. Issue 188-202. Doi: 10.1614/WS-D-1400035.1

Labrada, R. 1997. Problemas relacionados con el desarrollo del manejo de malezas en el mundo en desarrollo. pp. 7-13. En: FAO (ed.). Consulta de expertos en ecología y manejo de malezas. FAO, Roma.

Labrada, R. y C. Parker. 1996. El control de malezas en el contexto del manejo integrado de plagas. pp. 1-9. En: Labrada, R., J.C. Caseley y C. Parker (eds.). Manejo de malezas para países en desarrollo. FAO, Roma.

MADR (Ministerio de Agricultura y Desarrollo Rural). 2005. La cadena del banano en Colombia una mirada global de su estructura y dinámica 1991-2005. Documento de Trabajo No. 60. Ministerio de Agricultura y Desarrollo Rural, Observatorio Agrocadenas, Bogotá.

Martínez, A.M. y L. M. Hoyos. 2012. Banano (Musa AAA. Simmonds). pp. 349-369. En: Fischer, G. 
(ed.). Manual para el cultivo de frutales en el trópico. Produmedios, Bogotá.

Martins, D. y R. Pitelli. 1994. Interferência das plantas daninhas na cultura do amendoim das águas: efeitos de espaçamentos, variedades e períodos de convivência. Planta Daninha 12(2), 87-92. Doi: 10.1590/S0100-83581994000200006

Menalled, F. 2010. Consideraciones ecológicas para el desarrollo de programas de manejo integrado de malezas. Agroecología 5, 73-78.

Menalled, F., K. Gross y M. Hammond. 2001. Weed aboveground and seedbank community responses to agricultural management systems. Ecol. Appl. 11, 1586-1601. Doi: 10.2307/3061080

Monaco, T., S. Weller y F. Ashton. 2002. Weed science. Principles and practices. $4^{\text {th }}$ ed. John Wiley \& Sons, New York, NY.

Moreno, J. M., C. Blanco y R. Mendoza. 2009. Buenas prácticas agrícolas en el cultivo del banano en la región del Magdalena "Reducción del escurrimiento de plaguicidas al Mar Caribe". PNUMA/UCR/ CAR. Augura Medellín, Colombia.

Mortimer, M. 1997. La necesidad de los estudios sobre ecología de malezas para mejorar el manejo de malezas. pp.17- 26. En: FAO (ed.). Consulta de expertos en ecología y manejo de malezas. FAO, Roma.

Neve, P., M. Villa-Aiub y F. Roux. 2009. Evolutionarythinking in agricultural weed management. New Phytol. 184, 783-793. Doi: 10.1111/j.14698137.2009.03034.x

Nicholls, C. 2006. Bases agroecológicas para diseñar e implementar una estrategia de manejo de hábitat para control biológico de plagas. Agroecología 1, 37-48.

Oerke, E.C., H.W. Dehne, F. Schnbeck y A. Weber. 1994. Crop production and crop protection: Estimated losses in major food and cash crops. Elsevier Science, Amsterdam.

Owen, M. e I. Zelaya. 2005. Herbicide-resistant crop and weed resistance to herbicides. Pest Manage. Sci. 61, 301-311. Doi: 10.1002/ps.1015

Pimentel, D., S. McNair, J. Janecka, J. Wightman, C. Simmonds, C. O'connell, E. Wong, L. Russel, J. Zern, T. Aquino y T. Tsomondo. 2001. Economic and environmental threats of alien plant, animal, and microbe invasions. Agric. Ecosyst. Environ. 84, 1-20. Doi: 10.1016/S0167-8809(00)00178-X

Pinilla, C. y J. García. 2002. Manejo integrado de arvenses en plantaciones de banano (Musa AAA). pp. 222-235. En: Memorias XV Reunión Asociación de bananeros de Colombia (Acorbat). Augura, Cartagena, Colombia.

Plaza, G. A., M.D. Osuna, R. De Pardo y A. Heredia. 2006. Absorption and translocation of imazethapyr as a mechanism responsible for resistance of Euphorbia heterophylla L. biotypes to acetolactate synthase (ALS) inhibitors. Agron. Colomb. 24(2), 302-305.

Plaza, G. 2012. Manejo de malezas en frutales. pp. 238 251. En: Fischer, G. (ed.). Manual para el cultivo de frutales en el trópico. Produmedios, Bogotá.

PNUMA-UCR/CAR. 2012. REPCar Informe final del proyecto "Mejorando el Manejo de plaguicidas Agrícolas en Colombia, Costa Rica y Nicaragua". Experiencias del proyecto GEF reduciendo el escurrimiento de plaguicidas, Unidad de Coordinación Regional para el Caribe. Programa de las Naciones Unidad para el Medio Ambiente, Kingston, Jamaica.

Pollnac, F., L. Rew y F. Menalled. 2008. Spatial patterns, species richness and cover in weed communities of organic and conventional no-tillage spring wheat systems. Weed Res. 48, 398-407. Doi: 10.1111/j.1365-3180.2008.00631.x

Prameela, P. 2010. Identification of critical stages of weed competition and effect of weed competition in banana variety Palayankodan. J. Crop Weed 6(2), 59-62.

Radosevich, S.R., J.S. Holt y C.M. Ghersa. 1997. Weed ecology. Implications for management. $2^{\text {nd }} \mathrm{ed}$. John Wiley \& Sons, New York, NY.

Sabbatini, M.R., J.H. Irigoyen y M.N. Vernavá. 2004. Estrategias para el manejo integrado de malezas: problemática, resistencia a herbicidas y aportes de la biotecnología. pp 343-353. En: Echenique, V., C. Rubinstein y L. Mroginski (eds.). Biotecnología y mejoramiento vegetal. Instituto Nacional de Tecnología Agropecuaria (INTA), Buenos Aires.

Sangeetha, C. y P. Baskar. 2015. Allelopathy in weed management: A critical review. Afr. J. Agric. Res. 10(9), 1004-1015. Doi: 10.5897/AJAR2013.8434

Seeyave, J. y C.A. Phillips. 1970. The effect of weed competition on growth, yield and fruit quality of bananas. Pans 5(2), 343-347. Doi: 10.1080/09670877009411780

Schwartz, L., D. Gibson, K. Gage, J. Matthews, D. Jordan, M. Owen, D. Shaw, S. Weller, R. Wilson y B. Young. 2015. Seedbank and field emergence of weeds in glyphosate-resistant cropping systems in the United States. Weed Sci. 63, 425-439. Doi: 10.1614/WS-D-14-00089.1

Shaner, D. 2014. Lessons learned from the history of herbicide resistance. Weed Sci. 62(2), 427-431. Doi: 10.1614/WS-D-13-00109.1 
Smith, R., B. Maxwell, F. Menalled y L. Rew. 2006. Lessons from agriculture may improve the management of invasive plants in wildland systems. Front. Ecol. Environ. 4(8), 428-434. Doi: 10.1890/1540-9295(2006)4[428:LFAMIT]2.0. $\mathrm{CO} ; 2$

Smith, R, D. Mortensen y M. Ryan. 2010. A new hypothesis for the functional role of diversity in mediating resource pools and weed-crop competition in agroecosystem. Weed Res. 50, 37-48. Doi: 10.1111/j.1365-3180.2009.00745.x

Swanton, C., K. Harker y R. Anderson. 1993. Crop losses due to weeds in Canada. Weed Technol. 7, 537-542.

Terry, P.J. 1997. Manejo de malezas en frutales. Manejo de las malas hierbas en bananos y plátanos. pp. 327-332. En: Labrada, R., J.C. Caseley y C. Parker (eds.). Manejo de malezas para países en desarro1lo. FAO, Roma.

Vencill, W., R. Nichols, T. Webster, J. Soteres, C. Mallory-Smith, N. Burgos, W. Johnson y R. McClelland. 2012. Herbicide resistant: toward and understanding of resistance development and the impact of herbicide-resistance crops. Weed Sci. Spec. Isssue 2-30. Doi: 10.1614/WS-D-11-00206.1

Viloria, J. 2008. Banano y revaluación en el departamento del Magdalena 1997-2007. Documento de trabajo sobre economía regional 105. Banco de la República, Centro de Estudios Económicos Regionales (CEER). Cartagena, Colombia.

Ward, S.M., R.D. Cousens, M.V. Bagavathiannan, J.N. Barney, H.J. Beckie, R. Busi, A.S. Davis, J.S. Dukes, F. Forcella, R.P. Freckleton, E.R. Gallandt, L.M. Hall, M. Jasieniuk, A. Lawton-Rauh, E.A. Lehnhoff, M. Liebman, B.D. Maxwell, M.B. Mesgaran, J.V. Murray, P. Neve, M.A. Núñez, A. Pauchard, S.A. Queenborough y B.L. Webber. 2014. Agricultural weed research: a critique and two proposals. Weed Sci. 62, 672-678. Doi: 10.1614/WS-D-13-00161.1

Weston, L. y S. Duke. 2003. Weed and crop allelopathy. Crit. Rev. Plant Sci. 22 (3-4), 367-389. Doi: $10.1080 / 713610861$

Zamorano, C., H. López y G. Alzate. 2008. Evaluación de la competencia de arvenses en el cultivo de arveja (Pisum sativum) en Fusagasugá, Cundinamarca (Colombia). Agron. Colomb. 26(3), 443-450. 